\title{
Design and error compensation of active mechanically automated guided vehicle
}

\author{
Jianqiang Yan $^{1}, \mathrm{Mi} \mathrm{Li}^{1,2, *}$, Zhongxiang Chen ${ }^{1}$, and Yihang $\mathrm{Li}^{1}$ \\ ${ }^{1}$ Hunan Normal University, School of Engineering and Design, Changsha 410081, PR China \\ ${ }^{2}$ Central South University, School of Automation Changsha, Hunan 410083, PR China
}

Received: 15 June 2020 / Accepted: 10 February 2021

\begin{abstract}
To reduce the navigation control cost, this paper proposes a mechanical guidance control scheme that uses a cam-link mechanism as a steering control mechanism for an automated guided vehicle with a fixed driving path. According to the steering principle, a mathematical model of the steering system and the driving trajectory are established. By setting the boundary conditions, the vehicle trajectory is modeled using a quintic polynomial. The contour of the directional control cam is obtained based on the equation of the vehicle trajectory. Because errors occur in actual machining and assembly processes, errors will be classified based on their impact on the trajectory. The effects of various errors on the trajectory are quantitatively determined by using simulation methods with different parameters. Furthermore, an error compensation approach is designed to reduce the influence of the error on the trajectory directly or indirectly. Finally, experiment results illustrate that the adjustment accuracy of the proposed automated guided vehicle trajectory is $2 \mathrm{~mm}$.
\end{abstract}

Keywords: Mechanically guided trajectory / automated guided vehicle / cam curve algorithm / error compensation / cam link mechanism

\section{Introduction}

With the development of intelligent logistics equipment technologies, the automation of material handling has been greatly improved. Automatic guided vehicles are widely used in factories [1]. Almost all automated guided vehicles are based on electromagnetic or optical navigation control. Therefore, the electronic automatic guided vehicle has a high degree of flexibility. The driving route can be dynamically planned or modified according to actual needs [2]. However, the electromagnetic and optical navigation control vehicles need to use various sensors to sense the factory environment. And a complete electronic control system is required to the steering of the vehicle, which will lead to high costs [3-6]. In some cases, the vehicle only needs to follow a known fixed cycle trajectory. If the electronic navigation control scheme is still used, the manufacturing cost of the vehicle will increase. Although the mechanically guided vehicle cannot dynamically change its trajectory. But it has the characteristics of low production cost, strong robust and high reliability. Thus, in the fixed-cycle trajectory navigation control, it

\footnotetext{
* e-mail: 475431371@qq. com
}

has prominent advantages. In the fixed cycle trajectory navigation control, rail vehicles have been widely used. But it needs to install a fixed rail [7]. Therefore, it is of great significance to design an active mechanical navigation to control the vehicle.

One of the key technologies of active mechanical guidance process is to design a suitable mechanism to achieve automatic steering control. The mechanisms such as the space link mechanism [8], Geneva mechanism [9], cam mechanism [10], gear cam combination mechanism [11], and so on, can all serve as the steering mechanism. However, there is a non-linear relationship between the length of each link of the space link mechanism and the motion of the output shaft. And the analysis and synthesis of the space link mechanism is more complicated than the planar link mechanism [12]. Due to the poor dynamic performance of the Geneva mechanism, it is difficult to achieve high-precision control of the trajectory using this mechanism [13]. The cam mechanism only needs to design the cam profile according to the desired trajectory. When the desired trajectory changes, only the steering control cam needs to be replaced. At the same time, a fine adjustment mechanism can be used to correct the error of the cam guide mechanism. Therefore, it is feasible to realize the active mechanical guidance of the vehicle through the cam mechanism. 
Almost all traditional methods of cam profile curve are based on the principle of reversal method. The cam profile design is completed by setting various parameters such as the base circle radius of the cam, the movement law of the follower, and the movement angle. However, it has some limitations for the cams design with arbitrary desired trajectories. To overcome these problems, Zhou et al. proposed a design method of high-speed cam mechanism based on Fourier series [14]. But the Fourier series keep finite terms, and the theoretical design error always exists. Hseih presented a three-arc cam system design and analysis method based on the predecessors' results [15]. Flocker et al. established a closed-type modified trapezoidal cam motion function with adjustable positive and negative accelerations [16]. These previous works provide a good foundation for the design of steering cam with arbitrary trajectory.

The machining error of parts is inevitable in the manufacturing process. The accumulation of part machining errors will affect the assembly accuracy of the machine. To ensure the final assembly accuracy, proper assembly process methods need to be adopted during the assembly process. When the production volume is vast, the interchange method and the matching method are often used. When the final assembly accuracy of the machine is high and the quantity is small, repair and adjustment methods are more suitable. No matter which assembly method is used, it is necessary to analyze the effect of part machining error on assembly accuracy. Zhao et al. analyzed the influence of part machining error on the static return difference of the rotating vector reducer. And a mathematical model between the machining error of the parts and the static return difference is established. Based on the error analysis model, the distribution of parts manufacturing tolerances is completed [17]. Zhou proposed a method with a four-bar linkage to generate the desired curve. The accuracy of the generated curve is trimmed by adjusting the hinge position of the driven link [18]. Soong pointed out that the curve generated by the four-bar linkage can be adjusted by changing the length of the active link. In the method proposed by Soong, the desired curve was no longer limited by the number of precision control points [19]. To ensure the guiding accuracy of optical components, Dong designed an adjustment mechanism with slit diaphragm flexure [20]. Zhao analyzed the effect of link deviation and joint clearance on the angle error of the radar antenna deployment mechanism, and proposed a mechanism for adjusting the angle error [21]. Based on the DenavitHartenberg method and geometric closure conditions, Lin analyzed the accuracy of spatial multi-loop mechanism effected by paired bearings support joint clearance [22]. As a typical open-loop control system, the accumulation of errors will have a greater impact on the driving trajectory. Therefore, it is necessary to analyze the influence of parts manufacturing error on the accuracy of the driving trajectory.

In this paper, a cam lever mechanism is designed for the active mechanical guidance control scheme. First, a mathematical model of the steering system and driving trajectory is established. And the cam profile is obtained by the mathematical model. Second, simulation experiment is

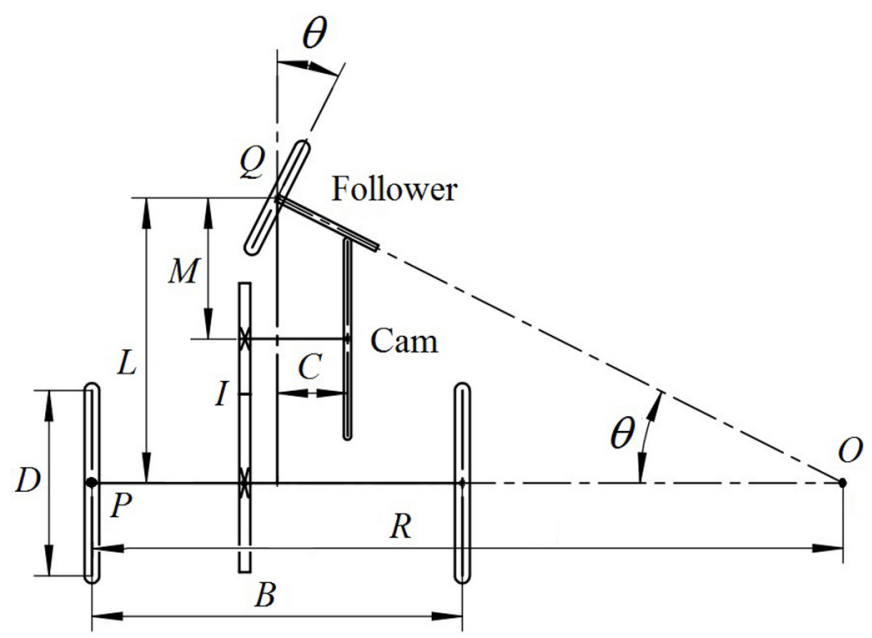

Fig. 1. Vehicle structure diagram.

used to quantitatively determine the effect of various errors on the trajectory separately and jointly. Finally, the error compensation mechanism and error adjustment strategy are given. The contributions of this paper are listed as follows.

- An active mechanical automatic guidance mechanism is proposed, which realizes electricity-free tracking control. The use of quintic polynomial in constructing the trajectory of the vehicle can be applied to the design of steering cam with arbitrary trajectory.

- The effect of the error on the trajectory is quantitatively analyzed and the error adjustment mechanism is given. The "V" type mechanism can be used to adjust the steering angle distribution errors. The spiral micrometer mechanism can be used to adjust the steering angle amplitude errors.

\section{Principles and mathematical models of active mechanical guidance}

For the cam mechanism, various motions can be realized by changing the cam profile. Therefore, in this paper, the cam swing lever mechanism is used as the steering control mechanism of the vehicle. The structure of the threewheeled mechanically guided vehicle is shown in Figure 1. Specifically, the front wheel is steering wheel and the other two wheels are driving wheels. The gear mechanism and the cam lever mechanism are placed between the rear and front wheels of the vehicle. Through the cam swing lever mechanism, the vehicle is controlled to travel on the desired trajectory. In this model, we set the wheelbase as $L$, the distance between the two rear wheels as $B$, the distance between the cam and the middle plane as $C$, the distance between the cam axis and the front axle as $M$, the diameter of the rear wheel is $\mathrm{D}$, the transmission ratio of the gear mechanism as $I$.

As shown in Figure 2, in order to evaluate the mechanical guidance scheme, the desired trajectory is set as a "Double 8" trajectory in this paper. The distance 


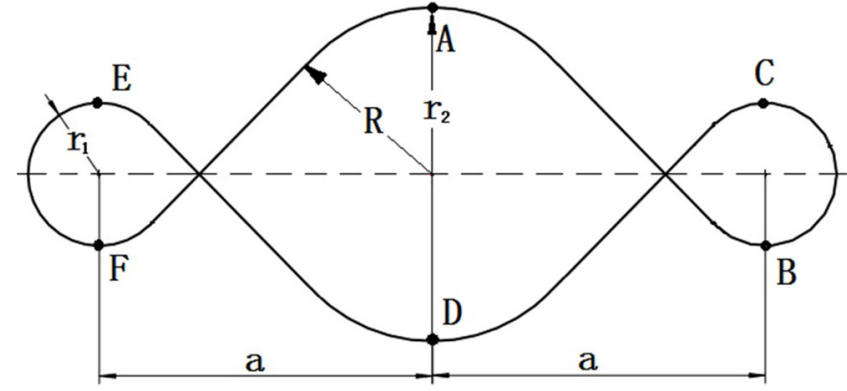

Fig. 2. Trajectory of vehicle.

between the center points of the two ends of the trajectory is $350 \mathrm{~mm}$. The trajectory is composed of left and right arcs and quintic polynomial curves connected with the two arcs. The trajectory $y=f(x)$ can be described by using the piecewise function as follows:

\section{See equation (1) below.}

where, $\mathrm{y}_{E F}, \mathrm{y}_{F A}, \mathrm{y}_{A B}, \mathrm{y}_{B C}, \mathrm{y}_{C D}, \mathrm{y}_{D E}$ are vertical coordinates of arcs $\widehat{E F}, \widehat{F A}, \widehat{A B}, \widehat{B C}, \widehat{C D}, \widehat{D E}$, respectively. $(a, 0)$ and $(-a, 0)$ are the circle centers of the right one and the left one, $r_{1}$ is the radius of arcs.

As shown in Figure 1, the turning radius of the midpoint $P$ at the left rear wheel is:

$$
\mathrm{R}=\frac{\mathrm{L}}{\tan \theta}+\mathrm{B} / 2
$$

where, $\theta$ is the steering angle of front wheel, $B$ is the distance between the two rear wheels, $L$ is the wheelbase of the vehicle.

Similarly, the cam radius can be expressed as:

$$
\mathrm{R}_{\mathrm{C}}=\mathrm{M}-\mathrm{C} * \tan \theta
$$

where, $M$ is the distance from the cam axis to the front axle, $C$ is the distance between the cam and the middle plane, and $\theta$ is the steering angle of front wheel.

According to the curvature radius formula, the curvature radius of the plane curve can be obtained:

$$
\mathrm{R}=\left|\frac{\left(1+y^{\prime 2}\right)^{\frac{3}{2}}}{y^{\prime \prime}}\right|, y^{\prime}=\frac{\mathrm{d} y}{\mathrm{~d} x}, y^{\prime \prime}=\frac{d^{2} y}{d x^{2}}
$$

where, $y=f(x)$ is the trajectory function of the vehicle.
With equations (2)-(4), the radius of cam can be evaluated:

$$
\mathrm{R}_{\mathrm{C}}=\mathrm{M}-\mathrm{C} * \frac{\mathrm{L}}{\mathrm{R}-\mathrm{B} / 2}
$$

According to equations (4) and (5), in order to avoid sudden changes in the cam profile, the trajectory function $y=f(x)$ should be continuous at each point. At the same time, the first and second derivative of the trajectory $\left(y^{\prime}\right.$ and $\left.y^{\prime \prime}\right)$ should also be continuous at each point. Therefore, by setting the boundary conditions of the quintic polynomial curve, the coefficients of each part of the curve are obtained.

Taking the $A B$ section coefficient as an example, the method for determining the coefficient of the quintic polynomial curve of each section is explained. Six boundary conditions should be set for six undetermined coefficients:

$$
\text { At } A \text { point, } x=0, y=r_{2}, y^{\prime}=0, y^{\prime \prime}=1 / r_{2}
$$

$$
\text { At } B \text { point, } x=a, y=-r_{1}, y^{\prime}=0, y^{\prime \prime}=1 / r_{1}
$$

Using the boundary conditions of points $A$ and $B$, it can be obtained according to equation (1):

$$
\begin{gathered}
\left(\begin{array}{cccccc}
1 & 0 & 0 & 0 & 0 & 0 \\
0 & 1 & 0 & 0 & 0 & 0 \\
0 & 0 & 2 & 0 & 0 & 0 \\
1 & \alpha & \alpha^{\wedge} 2 & \alpha^{\wedge} 3 & \alpha^{\wedge} 4 & \alpha^{\wedge} 5 \\
0 & 1 & 2^{*} \alpha & 3^{*} \alpha^{\wedge} 2 & 4^{*} \alpha^{\wedge} 3 & 5^{*} \alpha^{\wedge} 4 \\
0 & 0 & 2 & 6^{*} \alpha & 12^{*} \alpha^{\wedge} 2 & 20^{*} \alpha^{\wedge} 3
\end{array}\right)\left(\begin{array}{c}
\mathrm{C}_{0} \\
\mathrm{C}_{1} \\
\mathrm{C}_{2} \\
\mathrm{C}_{3} \\
\mathrm{C}_{4} \\
\mathrm{C}_{5}
\end{array}\right) \\
=\left(\begin{array}{c}
\mathrm{r}_{1} \\
0 \\
1 / \mathrm{r}_{1} \\
-\mathrm{r}_{1} \\
0 \\
1 / \mathrm{r}_{1}
\end{array}\right)
\end{gathered}
$$

i.e.,

$$
\begin{aligned}
& \mathrm{C}_{0}=\mathrm{r}_{1}, \quad \mathrm{C}_{1}=0, \quad \mathrm{C}_{2}=-\frac{1}{2 \mathrm{r}_{1}}, \quad \mathrm{C}_{3}=\frac{2}{\mathrm{r}_{1} \alpha}-\frac{20 \mathrm{r}_{1}}{\alpha^{3}}, \\
& \mathrm{C}_{4}=\frac{30 \mathrm{r}_{1}}{\alpha^{4}}-\frac{5}{2 \mathrm{r}_{1} \alpha^{2}}, \quad \mathrm{C}_{5}=\frac{1}{\mathrm{r}_{1} \alpha^{3}}-\frac{12 \mathrm{r}_{1}}{\alpha^{5}}
\end{aligned}
$$

$$
\begin{cases}y_{E F}= \pm \sqrt{r_{1}^{2}-(x+a)^{2}} & -\left(a+r_{1}\right) \leq x \leq-a \\ y_{F A}=C_{0}+C_{1} x+C_{2} x^{2}+C_{3} x^{3}+C_{4} x^{4}+C_{5} x^{5} & -a \leq x \leq 0 \\ y_{A B}=C_{0}+C_{1} x+C_{2} x^{2}+C_{3} x^{3}+C_{4} x^{4}+C_{5} x^{5} & 0 \leq x \leq a \\ y_{B C}= \pm \sqrt{r_{1}^{2}-(x-a)^{2}} & a \leq x \leq a+r_{1} \\ y_{C D}=-C_{0}-C_{1} x-C_{2} x^{2}-C_{3} x^{3}-C_{4} x^{4}-C_{5} x^{5} & 0 \leq x \leq a \\ y_{D E}=-C_{0}-C_{1} x-C_{2} x^{2}-C_{3} x^{3}-C_{4} x^{4}-C_{5} x^{5} & -a \leq x \leq 0\end{cases}
$$




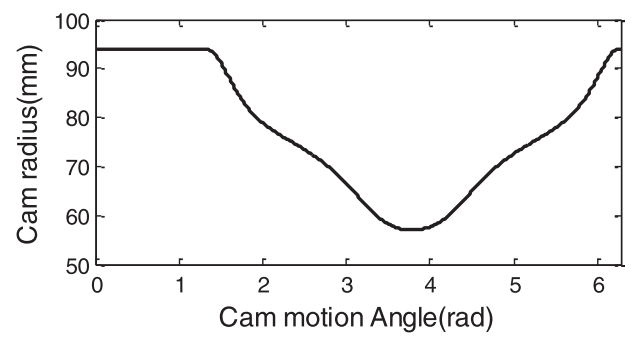

Fig. 3. Profile of directional control cam.

The distance traveled by the left rear wheel is the length of the trajectory line as shown in Figure 2. By using the cam profile algorithm described by equation (5), the cam profile under ideal conditions can be obtained. The cam profile is shown in Figure 3.

When the manufacturing and assembly errors of the parts are zero, the vehicle can follow the expected trajectory. However, in actual situations, various machining and assembly errors are inevitable. As a typical openloop control system, the mechanical guidance cannot implement real-time adjustment of errors according to the sensors, like the closed-loop control system. Due to the accumulation of errors, the travel trajectory will eventually deviate from the expected trajectory. Therefore, it is necessary to analyze the effect of errors on trajectory accuracy and design corresponding error compensation mechanisms.

\section{Error analysis and trajectory simulation}

\subsection{Error analysis}

As shown in Figure 1, the power input to the rear wheels is transmitted to the cam through gears. The steering follower connected with the front wheels is driven by the cam. The trajectory of the vehicle is controlled by the cam profile. When the front wheel turns according to the desired trajectory, the ideal trajectory can be obtained. However, in order to meet economic requirements, tolerances are included in the dimensions of the parts. The dimensional tolerance will cause the vehicle to fail to follow the desired trajectory. According to the structure of the vehicle, the assembly accuracy index required by the vehicle is the ratio of the front wheel angle to the displacement of the vehicle. The sources of errors that affect the assembly accuracy index are:

- Verticality error between steering follower and front wheel.

- Assembly error of the distance $\mathrm{C}$ from cam to the middle plane.

- Assembly error of the distance M from cam axis to front wheel axis.

- Assembly error of wheelbase L of vehicle.

- Machining error of directional control cam profile size.

- Machining error of diameter size of rear wheels.

- Accuracy error of transmission ratio I of rear wheel to cam mechanism.

To facilitate analysis, we set the left turning angle of front wheel as $\theta_{1}$, and the right turning angle as $\theta_{2}$, the
Table 1. Errors classification.

\begin{tabular}{ll}
\hline The sources of errors & Form of errors \\
\hline $\begin{array}{l}\text { 1. Verticality between steering } \\
\text { follower and front wheel }\end{array}$ & $\begin{array}{l}\text { Steering angle } \\
\text { distribution errors }\end{array}$ \\
$\begin{array}{l}\text { 1. Distance } C \\
\text { 2. Distance } M\end{array}$ & $\begin{array}{l}\text { Steering angle } \\
\text { 3. Wheelbase } L\end{array}$ \\
$\begin{array}{l}\text { 1. Cam profile size } \\
\text { 2. Diameter size of rear wheels }\end{array}$ & Composite errors \\
3. Transmission ratio $I$ & \\
\hline
\end{tabular}

amplitude of front wheel turning angle $A=\theta_{1}+\theta_{2}$. When the steering follower is not perpendicular to the front wheels, one of the left and right steering angles will always be greater than the theoretical value, and the other will always be less than the theoretical value. At the same time, the left and right steering angle errors are equal $\left(\Delta \theta_{1}=\Delta \theta_{2}\right)$. Therefore, the verticality only affects the distribution of the steering angle amplitude $A$, but does not affect the steering angle amplitude $A$. When the distance $C$ from the cam to the middle plane, the distance $M$ from the cam axis to the front wheel axis, and the wheelbase $L$ are greater than the theoretical value, the left and right turning angles will be less than the theoretical value. vice versa. Therefore, the distances $C, M$ and $L$ only affect the steering angle amplitude $A$, but do not affect the distribution of the steering angle amplitude $A$ between the left and right turning angles.

The cam profile error, as a shape profile tolerance, not only affects the steering angle amplitude $A$, but also affects the distribution of the steering angle amplitude $A$. Although the diameter of the rear wheels does not affect the steering angle, it will cause the ratio of the front wheel angle to the vehicle displacement to be not equal to the theoretical value. For example, when the diameter of the rear wheel is greater than the theoretical value, the length of the trajectory of the vehicle will be greater than the theoretical value. The transmission ratio between the rear wheel and the cam mechanism directly affects the assembly accuracy index. But when the gear mechanism is adopted, the transmission ratio of the involute gear transmission will not change due to the change of the center distance. Therefore, the effect of the transmission ratio error on the trajectory of the vehicle can be ignored. The effect of cam profile error and rear wheels diameter error can be reduced by improving machining accuracy.

According to the effect of error on steering angle, the errors can be classified into three types: (1) the steering angle distribution errors; (2) the steering angle amplitude errors; (3) the composite errors. The errors affecting the trajectory are classified as shown in Table 1.

\subsection{Trajectory simulation}

To determine the effect of the errors on the trajectory quantitatively, the "double 8" trajectory is set as the desired 
trajectory. The dimensions of the vehicle are $M=75.5 \mathrm{~mm}$, $C=29.5 \mathrm{~mm}, L=77 \mathrm{~mm}, B=100 \mathrm{~mm}$. Set the velocity of vehicle as $8 \mathrm{~m} / \mathrm{min}$ and the friction coefficient as 0.4 . The ratio between $R c$ and $R$ is shown in Figure 4, and the

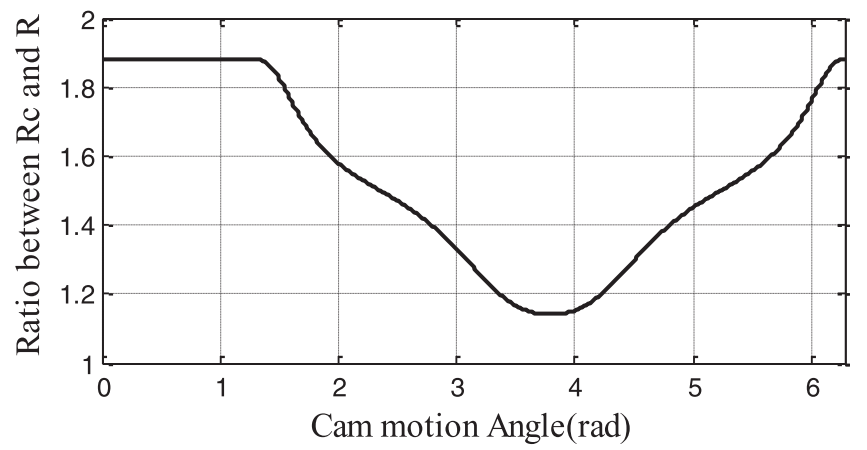

Fig. 4. The ratio between $R_{c}$ and $R$.

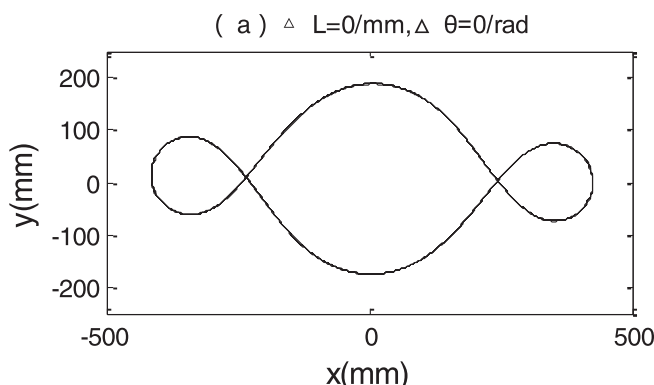

(b) $\Delta \mathrm{L}=0 / \mathrm{mm}, \Delta \theta=0.001 / \mathrm{rad}$

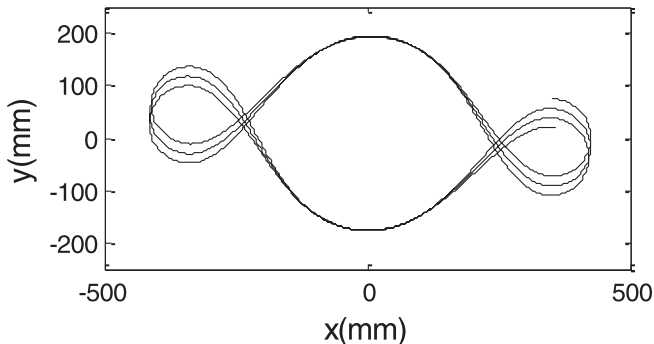

(c ) $\Delta \mathrm{L}=0 / \mathrm{mm}, \Delta \theta=0.002 / \mathrm{rad}$

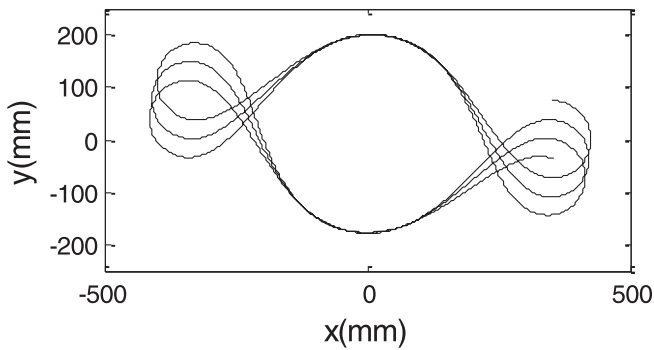

( d ) $\Delta L=0 / \mathrm{mm}, \Delta \theta=-0.001 / \mathrm{rad}$

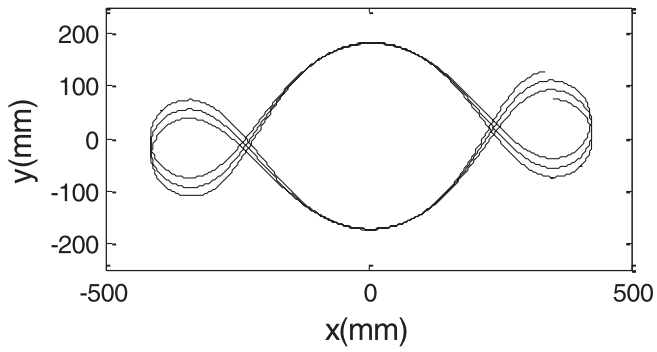

vehicle trajectory under ideal conditions is shown in Figure 5a.

Set the steering angle distribution error as $\Delta \theta$, the front wheel steering angle amplitude error as $\Delta L$. As shown in Figures $5 \mathrm{~b}$ to $4 \mathrm{~h}$, by setting $\Delta \theta$ and $\Delta L$ to different values, the vehicle trajectory under different errors can be obtained.

From Figures 5b to $5 \mathrm{~d}$, it is notable that even a small steering angle distribution error $\Delta \theta$ will cause a large deviation of the trajectory after the vehicle trajectory is repeated multiple times. Thus, the trajectory is extremely sensitive to the steering angle distribution error $\Delta \theta$, and the trajectory offset form is a rotational round the midpoint of the trajectory. When the steering angle distribution error $\Delta \theta$ is $0.001 \mathrm{rad}$, the trajectory offset after the vehicle travels one circle is as high as $20 \mathrm{~mm}$. When the steering angle distribution error $\Delta \theta$ is positive, the traveling trajectory is shifted clockwise around the midpoint of the trajectory.

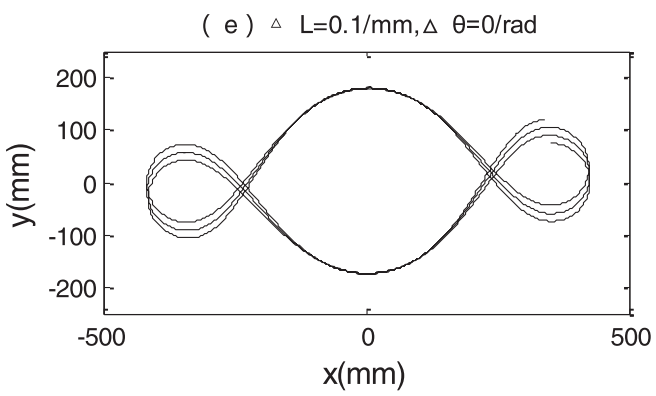

(f) $\Delta L=0.3 / \mathrm{mm}, \Delta \theta=0 / \mathrm{rad}$

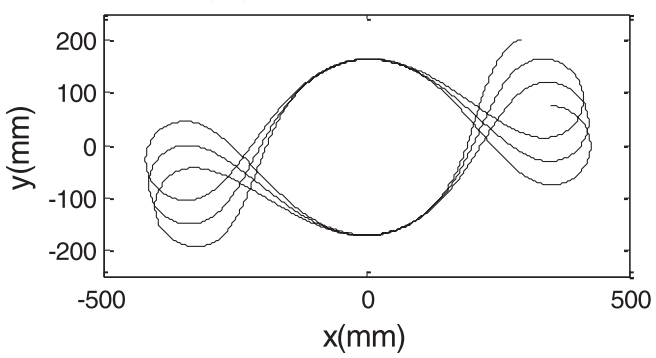

( g ) $\Delta \mathrm{L}=0.3 / \mathrm{mm}, \Delta \theta=-0.001 / \mathrm{rad}$

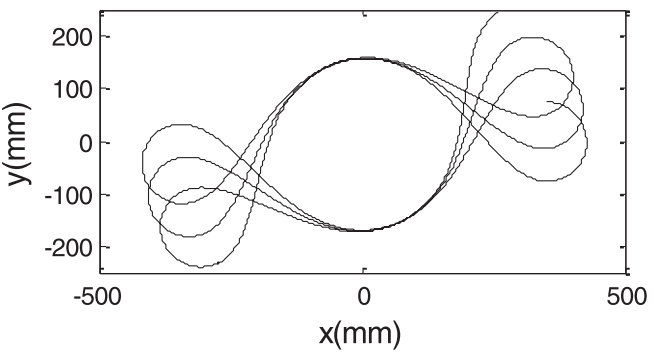

( h ) $\Delta \mathrm{L}=0.3 / \mathrm{mm}, \Delta \theta=0.001 / \mathrm{rad}$

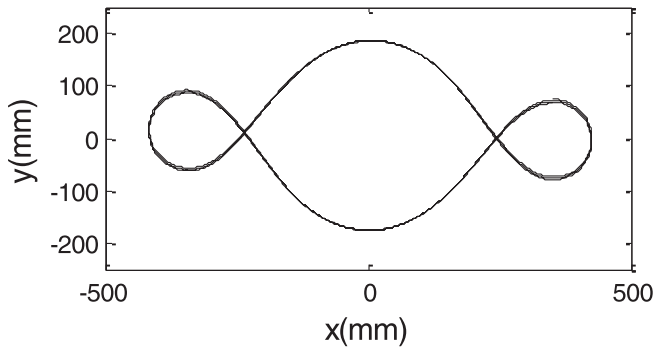

Fig. 5. Trajectory at different errors. 
When the steering angle distribution error $\Delta \theta$ is negative, the traveling trajectory is shifted counterclockwise around the midpoint of the trajectory.

It can be seen from Figures $5 \mathrm{e}$ to $5 \mathrm{f}$ that the trajectory offset of the vehicle caused by the steering angle amplitude error $\Delta L$ is similarly to the steering angle distribution error $\Delta \theta$, both of which are rotation offsets around the midpoint of the trajectory. But compared with the steering angle distribution error $\Delta \theta$, under the same trajectory offset error condition, its magnitude is 100 times. Thus, the steering angle amplitude error $\Delta L$ is insensitive.

As shown in Figures $5 \mathrm{~g}$ and $5 \mathrm{~h}$, when the steering angle distribution error $\Delta \theta$ and the steering angle amplitude error $\Delta L$ exist at the same time, the cumulative form of the errors generated by the two is determined according to the directions of the two. As shown in Figure $5 \mathrm{~g}$, when $\Delta \theta$ is negative and $\Delta L$ is positive, the trajectory errors generated by the two will accumulate in the same direction, and vice versa. In Figure $5 \mathrm{~h}$, although there are some errors in $\Delta \theta$ and $\Delta L$, the trajectory offset caused by the two can offset each other due to their different directions for the trajectory offset.

The impact of composite errors described in Table 1 can be reduced by improving machining accuracy. The other two kinds of errors are produced in manual assembly due to assembly conditions. It is more suitable to use the adjustment method to reduce. The steering angle distribution errors can be adjusted by using the vertical dimension of the steering lever and the front wheels as an adjustment ring. The steering angle amplitude errors can be adjusted by using the distance from the cam to the middle plane as an adjustment ring.

\section{Error adjustment mechanism and adjustment strategy}

In the manufacturing and assembly process, errors will inevitably occur due to insufficient processing and assembly conditions. In order to reduce the size of the error, the most direct way is to put forward higher requirements on the machining accuracy of the part. But this will increase manufacturing difficulty and processing costs. For the vehicle steering system, even small errors will have a great impact on the trajectory after error accumulation. Obviously improving the accuracy of parts processing is not the best solution. According to the simulation, the errors affecting the vehicle trajectory include steering angle distribution error and steering angle amplitude error. Therefore, we propose an adjustment strategy using fine-tuning mechanism to achieve error compensation.

\subsection{Steering angle amplitude adjustment mechanism}

In the cam swing lever steering mechanism, the distance $C$ from the cam to the middle plane directly affects the steering angle amplitude $A$. Therefore, the steering angle amplitude can be adjusted by changing the distance $C$. The relationship between distance $C$ and steering angle

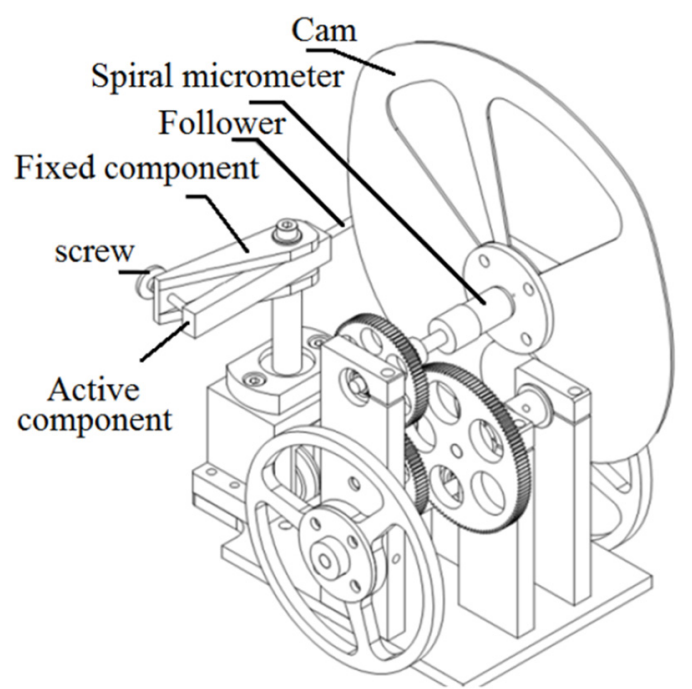

Fig. 6. Error adjustment mechanism.

amplitude $A$ can be expressed by equation (8).

$$
\Delta A=\tan ^{-1}\left(\frac{M-R_{c}}{C}\right)-\tan ^{-1}\left(\frac{M-R_{c}}{C+\Delta C}\right)
$$

where, $\Delta A$ is the change value of steering angle amplitude $A$, $\Delta C$ is the change value of distance $C, M$ is the distance from the cam axis to the front axle, $R_{\mathrm{c}}$ is the radius of cam.

According to the simulation, the vehicle trajectory error adjustment can be less than $2 \mathrm{~mm}$ when the division value adjusted by the distance $C$ is less than $0.013 \mathrm{~mm}$.

As shown in Figure 6, a spiral micrometer can be installed on the camshaft as an adjustment mechanism, and the division value of the spiral micrometer is $0.01 \mathrm{~mm}$. The spiral micrometer can adjust the axial displacement of the cam, thereby changing the steering angle amplitude. When the distance is increased, the amplitude of the steering angle amplitude decreases. While the steering angle amplitude will increase with the decrease of the distance.

\subsection{Steering angle distribution adjustment mechanism}

According to the simulation, the vehicle trajectory error adjustment can be less than $2 \mathrm{~mm}$ when the steering angle distribution adjustment division value is less than 1e-4rad.

As shown in Figure 6, the steering angle distribution adjustment mechanism is composed of a fixed component, a movable component, and a screw. The fixed component is rigidly connected to the front wheel vertical shaft, and the movable component is sleeved on the front wheel vertical shaft. The angle between the two is changed by screws. Setting the length of the fixed component as $L_{1}$, the length of the adjustment screw as $L_{2}$, the screw pitch as $q$, and the number of knobs on the end of the screw as $Z$, then we can obtain:

$$
\Delta \theta=\tan ^{-1}\left(\frac{L_{2}}{L_{1}}\right)-\tan ^{-1}\left(\frac{L_{2}-q / Z}{L_{1}}\right)
$$




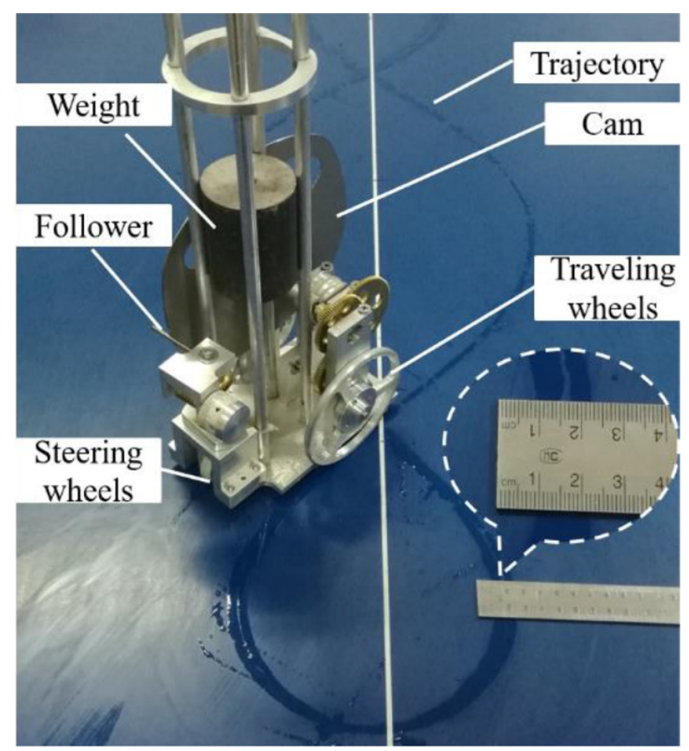

Fig. 7. "Double 8" trajectory vehicle experiment.

In this study, the length of the fixed component is $L_{1}=50 \mathrm{~mm}$, the length of the adjustment screw is $L_{2}=10 \mathrm{~mm}$, the screw pitch is $q=0.25 \mathrm{~mm}$, and the number of knob scales is $Z=50$, then $\Delta \theta=9.6 \mathrm{e}-5 \mathrm{rad}$.

\subsection{The adjustment strategy}

If the vehicle trajectory deviates around a certain center of the trajectory, the vehicle trajectory can be roughly adjusted by the steering angle distribution adjustment mechanism until the vehicle trajectory offset decreases. Because the vehicle trajectory steering angle distribution error $\Delta \theta$ is extremely sensitive, it is difficult to attempt to correct and adjust the vehicle trajectory offset error only through the steering angle distribution fine-tuning mechanism. The vehicle trajectory offset is not sensitive to the steering angle amplitude error $\Delta C$, and the steering angle amplitude error $\Delta C$ and the steering angle distribution error $\Delta \theta$ can compensate each other.

\section{Experiment}

In order to verify the proposed cam profile and the error adjustment mechanism, two subjects with different desired trajectories are presented in this section.

In the first case, the desired trajectory is set to the "double 8" trajectory, as shown in Figure 2. The coordinates of points A, B, C, D, E, F are $(0,180),(350,-70),(350,70)$, $(0,-180),(-350,70),(-350,-70)$, respectively. The vehicle is required to pass through all precision points in sequence according to the "double 8" trajectory. The coincidence degree of the cyclic trajectory of the vehicle is used as the evaluation index. Using the method proposed in the article, the profile of directional control cam is obtained. The active mechanical guided vehicle is as shown in Figure 7. Put the vehicle on the table tennis table and record the actual trajectory by hanging an ink drop

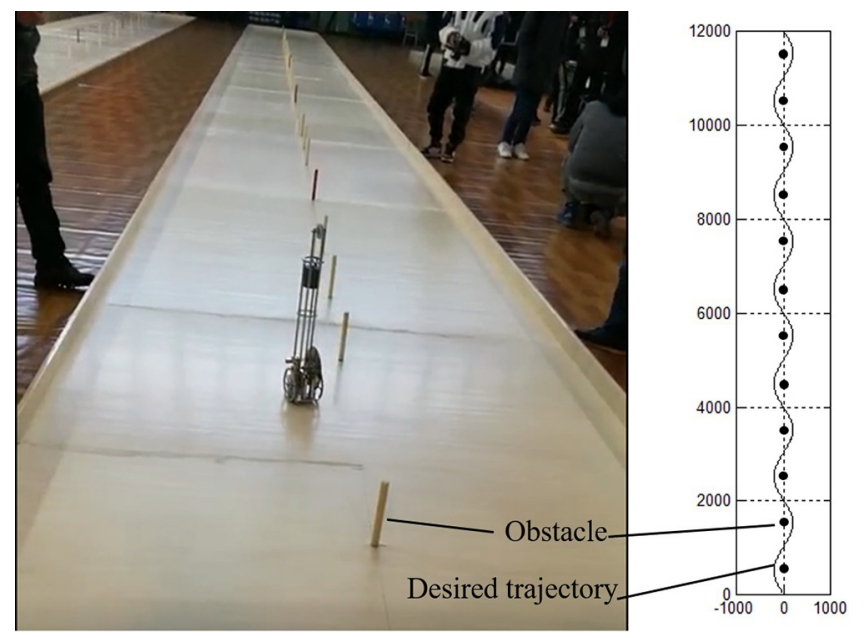

Fig. 8. "S" trajectory vehicle experiment.

device on the vehicle. The moving energy of the vehicle is transformed from the gravitational potential energy of the $1 \mathrm{Kg}$ weight suspended on the vehicle. The velocity of vehicle we measured in experiment is $7.4 \mathrm{~m} / \mathrm{min}$. Experimental results show that the trajectory error produced by the vehicle after 20 cycles is $10 \mathrm{~mm}$. However, it should be noted that the error in different place is non-uniform, the reason is that there are steering angle distribution errors and steering angle amplitude errors in the vehicle. The traveling trajectory is shifted clockwise around the midpoint of the trajectory. The positions with the largest error in the trajectory are points B, C, E and F, as shown in Figure 2.

In the second case, the desired trajectory is set to the " $\mathrm{S}$ " trajectory, as shown in Figure 8. There are many obstacles arranged in the traveling direction of the vehicle. The distance of these obstacles is $1000 \mathrm{~mm}$, and the diameter is $20 \mathrm{~mm}$. The vehicle is required to follow the "S" trajectory and bypass all obstacles in turn. The number of obstacles that the vehicle can successfully bypass is used as an evaluation indicator. Experimental results show that the vehicle can successfully bypass 22 obstacles.

\section{Conclusion}

This paper proposed a design scheme that uses a cam swing lever as the direction control mechanism. A mathematical model of the trajectory was established using a quintic polynomial, and a directional control cam profile algorithm was proposed based on the formula of curvature radius. For any motion trajectory in the plane, the corresponding cam profile can be obtained by the algorithm proposed in this paper.

By analyzing the impact of the vehicle error on the driving trajectory, it is found that the impact of the manufacturing error and assembly error can be converted into the steering angle distribution error and the steering angle amplitude error. The steering angle distribution error and the steering angle amplitude error can compensate each other. Therefore, in the design of the vehicle error 
adjustment mechanism, only one kind of error adjustment mechanism needs to be added to complete the vehicle trajectory error adjustment.

Although this article has completed the design of the steering mechanism and error compensation of the vehicle, there are still large differences from the actual factory application environment. Due to the accumulation of errors, trajectory errors are still inevitable. Therefore, how to realize the dynamic correction of the trajectory error based on the minimum detection signal is the content of subsequent research.

\section{References}

[1] T. Leanh, M.B. Koster, A review of design and control of automated guided vehicle systems, European Journal of Operational Research 171, 1-23 (2006)

[2] J. Long, C.L. Zhang, The summary of AGV guidance technology, Advanced Materials Research 591, 1625-1628 (2012)

[3] P. Yin, W. Li, Y. Duan, Combinatorial inertial guidance system for an automated guided vehicle, 2018 IEEE 15th International Conference on Networking, Sensing and Control 1, 1-6 (2018)

[4] S. Lee, H. Yang, Navigation of automated guided vehicles using magnet spot guidance method, Robotics and Computer-integrated Manufacturing 28, 425-436 (2012)

[5] S. Zeng, R. Xue, Y. Wang, The Research of AGV Guidance Systems Based on RFID Positioning, Computational Science and Engineering 1, 99-104 (2016)

[6] M.J. Seelinger, J. Yoder, Automatic visual guidance of a forklift engaging a pallet, Robotics and Autonomous Systems 54, 1026-1038 (2006)

[7] C. Martina, P. Alessandro, S. Fabio, Modelling of rail guided vehicles serving an automated parts-to-picker system, IFACPapersOnLine 51, 1476-1481 (2018)

[8] C. Chen, J. Angeles, Generalized transmission index and transmission quality for spatial linkages, Mechanism and Machine Theory 42, 1225-1237 (2007)

[9] J.J. Lee, K.F. Huang, Geometry analysis and optimal design of Geneva mechanisms with curved slots, Proceedings of the Institution of Mechanical Engineers, Part C: Journal of Mechanical Engineering Science 218, 449-459 (2004)
[10] E. Ottaviano, D. Mundo, G.A. Danieli, Numerical and experimental analysis of non-circular gears and cam-follower systems as function generators, Mechanism and machine theory 43, 996-1008 (2008)

[11] F.L. Litvin, P.I. Gonzalez, A. Fuentes, Tandem design of mechanisms for function generation and output speed variation, Computer Methods in Applied Mechanics and Engineering 198, 860-876 (2009)

[12] C.H. Suh, Design of space mechanisms for function generation, Journal of Engineering for Industry $\mathbf{9 0}$, 507-512 (1968)

[13] M. Heidari, M. Zahiri, H. Zohoor, Optimization of kinematic characteristic of geneva mechanism by genetic algorithm, World Academy of Science: Engineering and Technology 44, 387-395 (2008)

[14] C. Zhou, B. Hu, S. Chen, Design and analysis of high-speed cam mechanism using Fourier series, Mechanism and Machine Theory 104, 118-129 (2016)

[15] J.F. Hsieh, Design and analysis of cams with three circulararc profiles, Mechanism and Machine Theory 45, 955-965 (2010)

[16] F.W. Flocker, A versatile cam profile for controlling interface force in multiple-dwell cam-follower systems, Journal of Mechanical Design 134, 094501 (2012)

[17] H. Zhao, M. Wang, L.L. Zhang, Static backlash analysis and study on error distribution of RV reducer, Journal of Tianjin University (Science and Technology) 49, 164-170 (2016)

[18] H. Zhou, Synthesis of adjustable function generation linkages using the optimal pivot adjustment, Mechanism and Machine Theory 44, 983-990 (2009)

[19] R.C. Soong, S.B. Chang, Synthesis of function-generation mechanisms using variable length driving links, Mechanism and Machine Theory 46, 1696-1706 (2011)

[20] S.Z. Dong, K. Guo, X.L. Li, Design and analysis of adjustment mechanism with slit diaphragm flexures for optical elements, Chinese Optics 10, 790-797 (2017)

[21] Q. Zhao, J. Guo, J. Hong, Analysis of angular errors of the planar multi-closed-loop deployable mechanism with link deviations and revolute joint clearances, Aerospace Science and Technology 87, 25-36 (2019)

[22] F. Lin, C. Chen, J. Chen, Accuracy analysis of spatial multiloop mechanism effected by paired bearings support joint clearance, Journal of Mechanical Science and Technology 34, 1-17 (2020)

Cite this article as: J. Yan, M. Li, Z. Chen, Y. Li, Design and error compensation of active mechanically automated guided vehicle, Mechanics \& Industry 22, 14 (2021) 